\title{
Hartmann's procedure and laparoscopic reversal versus primary anastomosis and ileostomy closure for left colonic perforation
}

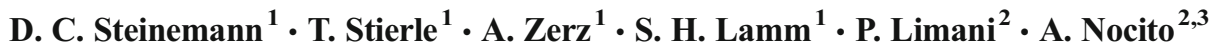

Received: 16 March 2015 / Accepted: 16 June 2015 / Published online: 26 June 2015

(C) Springer-Verlag Berlin Heidelberg 2015

\begin{abstract}
Purpose Emergency surgical strategies for acute left-sided colonic perforation are evolving preferring primary anastomosis (PA) with ileostomy to Hartmann's procedure (HP) based on the morbidity and reversal rates. However, HP is still commonly performed. Hartmann's reversal is associated with considerable morbidity. It is of interest whether laparoscopic reversal results in a lower morbidity as retrospective data suggest. Here, we compared the combined morbidity rates for two surgical strategies: strategy A, HP followed by laparoscopic reversal, and strategy B, sigmoid resection with PA followed by ileostomy closure.

Methods Prospectively collected data of all consecutive patients undergoing HP for benign left-sided colonic perforation between 2010 and 2014 were retrospectively compared to data of patients undergoing PA. Groups were matched for age and Charlson comorbidity index. Additionally, patients were analyzed for American Society of Anesthesiologists score, body mass index, and peritonitis stage. End points were morbidity, operation time, reversal rate, time to reversal, and length of hospital stay.
\end{abstract}

This study has been orally presented at the 14th World Congress of Endoscopic Surgery in Paris on June 2014.

D. C. Steinemann and T. Stierle contributed equally to this work.

D. C. Steinemann

daniel.steinemann@ksbl.ch

1 Department of Surgery, Cantonal Hospital Baselland, Bruderholz, CH-4101 Bruderholz, Switzerland

2 Division of Visceral and Transplantation Surgery, University Hospital Zurich, Raemistrasse 100, CH-8091 Zürich, Switzerland

3 Department of Surgery, Cantonal Hospital Baden, CH-5404 Baden, Switzerland
Results The study included 32 patients for whom Hartmann's reversal was planned, along with 32 matched patients who underwent PA and diverting ileostomy. Median age was 75 and 72 years, Charlson score was 6 (4-9) and 6 (5-7), and patients classified by the American Society of Anesthesiologists (ASA) higher than III were $81 \%$ in both groups. Combined major morbidity rates were $21 \%$ for strategy $\mathrm{A}$ and $20 \%$ for strategy B $(p=1.0)$. Combined comprehensive complication index was $16.4 \pm 14.1$ and $12.3 \pm 19.1(p=0.08)$. HP reversal by laparoscopy was achieved in $71 \%$. The colostomy reversal rate was $75 \%$ compared to ileostomy closure rate of $88 \%(p=0.34)$.

Conclusions Laparoscopic Hartmann's reversal is achievable in a high proportion of patients. Strategy B tends to have lower overall morbidity; meanwhile, major morbidity seems to be similar. Yet, in critically ill patients and in the absence of expertise of the surgeon on call, HP followed by elective laparoscopic reversal represents a viable alternative.

Keywords Diverticulitis · Hartmann · Primary anastomosis · Colon perforation $\cdot$ Laparoscopic reversal

\section{Introduction}

Left colonic perforation with generalized purulent or fecal peritonitis is a life-threatening condition that occurs with an annual incidence of 3.4 to 4.5 per 100,000 individuals [1]. Its most common etiology is acute diverticulitis, and it is frequently also the first manifestation of diverticular disease [1]. Perforated diverticulitis has been occurring with growing frequency, predominantly among elderly patients with multiple comorbidities [2]. In this population, the necessary emergency surgical treatment is associated with substantial morbidity and mortality [3]. 
Hartmann's procedure (HP) was originally developed for malignant colonic obstruction in 1923 and has generally been considered the gold standard for emergency treatment of acute perforated diverticulitis [4]. However, Hartmann's reversal is associated with a high morbidity rate of $20-50 \%$ and a mortality rate of up to $5 \%$. Therefore, reversal procedures are frequently denied to a majority of multimorbid patients, leading to permanent colostomy rate of $35-56 \%$ in these cases [5-7]. Consequently, surgical management of left-sided colonic perforation is still controversially discussed $[1,3,8]$. A number of retrospective studies [8-11] and three systematic reviews [12-14] have reported that primary anastomosis (PA) with ileostomy has a lower mortality than HP. However, mortality is reportedly comparable within a subgroup of high-risk patients [12-14], thus suggesting a selection bias in favor of younger and healthier patients with lower peritonitis scores in the primary anastomosis group [13-15]. A recent casematched study [11] and two multicenter randomized controlled trials $[16,17]$ also demonstrated no difference in overall morbidity and mortality between HP and PA.

Laparoscopic lavage, which has been recently proposed for colonic perforation with purulent peritonitis $[18,19]$, is associated with a high-failure rate among patients with severe peritonitis and multiple comorbid conditions [20, 21]. However, prospective randomized trials are still ongoing. Therefore, in daily clinical practice, many surgeons still opt for a HP $[3,22]$.

The laparoscopic Hartmann's reversal (LHR) appears to be a promising alternative to open surgery with data showing benefits regarding morbidity, postoperative recovery, reoperation rates, and length of hospital stay [5, 23-26]. A comparison of LHR and open Hartmann reversal in 4148 patients out of the American College of Surgeons National Surgical Quality Improvement Program database showed a significant lower overall complication rate in favor of the laparoscopic approach (18.4 versus $27 \%, p<0.0001)$ [27]. However, the literature lacks well-designed studies comparing open to laparoscopic Hartmann's reversal. Moreover, the available data are likely to be confounded by a selection bias in favor of younger and healthier patients receiving LHR.

The present study used a case-match approach with the aim of comparing two strategies, i.e., the combined morbidity of HP followed by LHR (strategy A) to that of sigmoid resection with PA and diverting ileostomy followed by ileostomy closure (IC; strategy B) in patients with benign left-sided colonic perforation and generalized peritonitis.

\section{Material and methods}

This study included all consecutive patients who underwent HP for benign left-sided colonic perforation at the Cantonal Hospital Baselland, considered a secondary center hospital, between August 2010 and March 2014. These patients were compared to a matched cohort of patients who underwent PA with protective ileostomy for benign colonic perforation at the University Hospital Zurich. General surgeons on call performed HP, senior laparoscopic surgeons performed LHR, and gastrointestinal surgeons performed PA.

All included patients were matched for age and comorbidity according to the Charlson comorbidity score (Table 1) [28]. Patients were also compared with regard to baseline characteristics, including the American Society of Anesthesiologists (ASA) score, body mass index (BMI), and stage of peritonitis according to the Hinchey classification (Table 1) [29].

Cumulative postoperative major morbidity of strategy A (HP followed by LHR) compared to strategy B (sigmoid resection with PA and diverting ileostomy followed by ileostomy closure (IC)) was assessed according to the Clavien-Dindo classification (Table 1), with a major surgical complication defined as grade IIIb or more [30]. We also quantified the overall morbidity of both therapeutic strategies using the comprehensive complication index (CCI) [31], a continuous scale that cumulates all postoperative complications. Furthermore, postoperative death within 30 days after surgery, operation time, reversal rate, time to reversal, and length of hospital stay were assessed. Patients were analyzed in an intention-to-treat manner.

\section{Statistical analysis}

Analyses of descriptive statistics and of significant differences were performed using $\mathrm{GraphPad}^{\circledR}$ Prism version 5.00 for Windows (GraphPad Software, San Diego, CA, USA). Proportions were compared between groups using a two-tailed Mann-Whitney test assuming a nonparametric distribution. Categorical variables were compared using a two-sided Fisher's exact test. The level of significance was set at 0.05 .

\section{Surgical technique of strategy A: Hartmann's procedure followed by laparoscopic Hartmann's reversal}

HP involved resection of the involved rectosigmoid - or the left hemicolon if necessary - through a midline laparotomy. The rectal stump was closed using a Contour ${ }^{\mathrm{TM}}$ curved cutter stapler (2.5 in/64 mm; Ethicon Endo-Surgery, Somerville, NJ, USA). No omentoplasty was performed. A left colostomy was performed, without mobilization of the splenic flexure. Peritoneal irrigation was performed prior to laparotomy closure.

LHR was planned for all patients. Prior to colostomy closure, all patients underwent colonoscopy and contrast enema examination of the colon and the rectal stump.

LHR was preceded by mechanical bowel preparation with Moviprep $^{\mathrm{TM}}$ (Norgine B.V., Amsterdam, The Netherlands). After administration of single-shot antibiotic prophylaxis with cefazolin and metronidazole, the colostomy was mobilized 
Table 1 (a) Charlson comorbidity index [27], (b) Clavien-Dindo classification [29], and (c) Hinchey classification [28]

(a) Charlson comorbidity index

Condition

Myocardial infarction

Congestive heart failure

Peripheral vascular disease

Cerebrovascular disease

Dementia

Chronic pulmonary disease

Connective tissue disease

Peptic ulcer disease

Mild liver disease

Diabetes without end-organ damage

Hemiplegia

Moderate or severe renal disease

Diabetes with end-organ damage

Tumor without metastases

Leukemia

Lymphoma

Moderate or severe liver disease

Metastatic solid tumor

Acquired immune deficiency syndrome

Age: for each decade, $>40$ years of age

(b) Clavien-Dindo classification

Grade

Minor complications

I

II

III

a

Major complications

IV

$\mathrm{V}$

(c) Hinchey classification

Grade

I

II

III

IV
Score

1

2

3

6

1

Definition

Any deviation from the normal postoperative course without the need for pharmacological treatment or surgical, endoscopic, and radiological interventions

Allowed therapeutic regimens are as follows: drugs as antiemetics, antipyretics, analgesics, diuretics, and electrolytes and physiotherapy. This grade also includes wound infections opened at the bedside

Requiring pharmacological treatment with drugs other than such allowed for grade I complications

Blood transfusions and total parenteral nutrition are also included

Requiring surgical, endoscopic, or radiological intervention

Intervention not under general anesthesia.

Intervention under general anesthesia

Life-threatening complication (including cerebral nervous system complications as brain hemorrhage, ischemic stroke,

subarachnoidal bleeding, but excluding transient ischemic attacks).

Requiring intermediate or intensive care unit management

Single-organ dysfunction (including dialysis)

Multi-organ dysfunction

Death of patient

Definition

Localized abscess (paracolonic)

Pelvic abscess

Purulent peritonitis (the presence of pus in the abdominal cavity)

Feculent peritonitis (fecal contamination of the abdominal cavity) from the abdominal wall. Resection of the colostomy-bearing colonic segment was performed before introduction of the anvil of a 31-mm circular stapler $\left(\right.$ EEA $^{\mathrm{TM}} 3.5 / 31 \mathrm{~mm}$; Covidien plc, Dublin, Ireland) and repositioning of the colon 
into the abdominal cavity. For laparoscopic reversal, a 12-mm trocar (Versastep ${ }^{\mathrm{TM}}$; Covidien plc, Dublin, Ireland) was inserted through the former colostomy site, and the fascia was closed around it. After pneumoperitoneum establishment, three additional 5-mm trocars (Karl Storz, GmbH \& Co KG, Tuttlingen, Germany) were placed step-by-step under vision in a diamond position. When needed, an additional $5-\mathrm{mm}$ trocar was placed epigastrically in the midline. After adhesiolysis, the left colon was mobilized, including the splenic flexure and the rectal stump. End-to-end colorectal anastomosis was performed using a 31-mm circular stapler. An air leak test was performed.

\section{Surgical technique of strategy B: sigmoid resection with primary anastomosis and diverting ileostomy followed by ileostomy closure}

Emergency sigmoid or left colonic resection with PA was performed through a midline incision, followed by identification and resection of the perforated and diverticula-bearing colonic segment. Mobilization of the left colon and the splenic flexure, including ligation of the inferior mesenteric vessels, were performed to create tension-free transanally stapled colorectal anastomosis (CDH 29 mm; Ethicon Endo-Surgery, Somerville, NJ, USA). Anastomosis integrity was verified by an air leak test. Peritoneal lavage and abdominal drain insertion were routinely employed. To create a diverting ileostomy, a loop of terminal ileum was brought to the skin in the left lower abdomen. The time to reversal of loop ileostomy was determined according to the patient's clinical condition. Ileostomy reversal was performed by mobilization of the stoma entry and end-to-end sutured anastomosis with or without resection of the stoma-bearing ileum segment. The skin wound was drained and primarily closed.

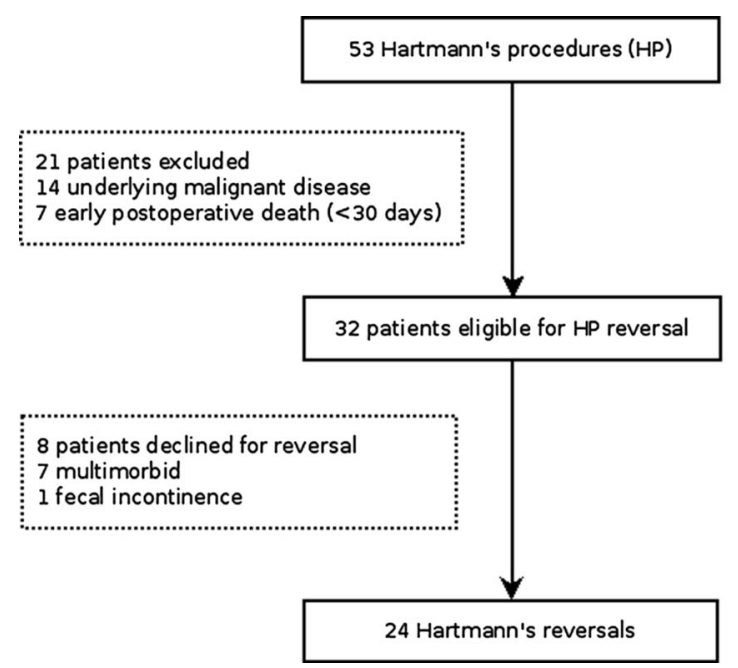

\section{Antibiotic treatment}

All patients received appropriate antibiotic treatment after the primary resectional procedure (amoxicillin/ clavulanic acid or tazobactam; in cases of pencillin intolerance, ciprofloxacin and metronidazole). Single-shot antibiotic prophylaxis (2nd generation cephalosporin and metronidazole) was administered prior to the reversal procedure.

\section{Results}

\section{Patient selection}

Fifty-three patients presenting with acute left-sided colonic perforation and generalized purulent or fecal peritonitis underwent HP (Fig. 1). Fourteen patients with underlying malignant disease and 7 patients who died in the postoperative course ( $<30$ days after surgery) after HP were excluded from the study. The remaining 32 patients were included in the analysis (strategy A).

\section{Patient characteristics of matched cohorts}

Patients who underwent strategy A were matched to 32 patients who underwent strategy B. Table 2 shows median age, median Charlson comorbidity index, gender, BMI, and ASA score, which were comparable between groups, with a tendency for a higher proportion of ASA IV patients undergoing strategy A (31.3 vs. $9.4 \%, p=0.06)$. Among the 32 patients who underwent HP, 21 presented with acute complicated diverticulitis while the remaining 11 patients had left colonic perforation with generalized peritonitis from other causes of perforation (Table 2).

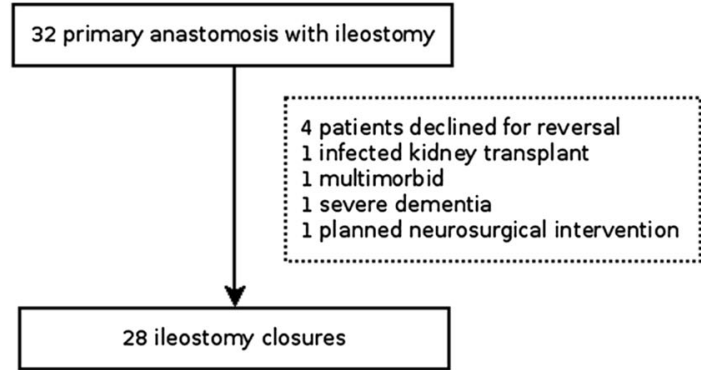

Fig. 1 Consort diagram 
Table 2 Patient characteristics. Major morbidity is defined as Clavien-Dindo score $\geq$ IIIlb

\begin{tabular}{|c|c|c|c|}
\hline & Strategy A $(n=32)$ & Strategy B $(n=32)$ & $p$ value \\
\hline Median age in years (range) & $75(67-83)$ & $72(64-81)$ & 0.38 \\
\hline Median Charlson score (range) & $6(4-9)$ & $6(5-7)$ & 0.94 \\
\hline Gender, men/women & $15 / 17$ & $12 / 20$ & 0.61 \\
\hline Median BMI (range) & $26(22-31)$ & $25(22-28)$ & 0.72 \\
\hline $\mathrm{ASA} \geq \mathrm{III}, n(\%)$ & $26 / 32(81.3 \%)$ & $26 / 32(81.3 \%)$ & 1.0 \\
\hline ASA I & $0 / 32(0 \%)$ & $0 / 32(0 \%)$ & 1.0 \\
\hline ASA II & $6 / 32(18.3 \%)$ & $6 / 32(18.3 \%)$ & 1.0 \\
\hline ASA III & $16 / 32(50 \%)$ & $23 / 32(71.9 \%)$ & 0.13 \\
\hline ASA IV & $10 / 32(31.3 \%)$ & $3 / 32(9.4 \%)$ & 0.06 \\
\hline \multicolumn{4}{|l|}{ Diagnosis } \\
\hline Diverticulitis, $n(\%)$ & $21 / 32(65.7 \%)$ & $27 / 32(84.4 \%)$ & \\
\hline Other perforation, $n(\%)$ & $4 / 32(12.5 \%)$ & $0 / 32(0.0 \%)$ & \\
\hline Anastomotic leakage, $n(\%)$ & 4/32 (15.2\%) & $3 / 32(9.4 \%)$ & \\
\hline Colon ischemia, $n(\%)$ & $3 / 32(9.4 \%)$ & $2 / 32(6.3 \%)$ & \\
\hline \multicolumn{4}{|l|}{ Peritonitis } \\
\hline Hinchey III/IV & $19 / 13$ & $25 / 7$ & 0.10 \\
\hline \multicolumn{4}{|l|}{ Morbidity } \\
\hline Combined major morbidity (\%) & $12 / 56(21.4 \%)$ & $12 / 60(20 \%)$ & 1.0 \\
\hline Combined CCI, mean $\pm \mathrm{SD}$ & $16.4 \pm 14.1$ & $12.3 \pm 19.1$ & 0.08 \\
\hline Resection major morbidity (\%) & $10 / 32(31.3 \%)$ & $8 / 32(25 \%)$ & 0.78 \\
\hline Resection CCI, mean \pm SD & $23.6 \pm 23$ & $16.8 \pm 20.7$ & 0.25 \\
\hline Reversal major morbidity (\%) & $2 / 24(8.3 \%)$ & $4 / 28(14.3 \%)$ & 0.67 \\
\hline Reversal CCI, mean \pm SD & $12.2 \pm 19.9$ & $8.8 \pm 20.5$ & 0.33 \\
\hline Reversal rate, $n(\%)$ & $24 / 32(75.0 \%)$ & $28 / 32(87.5 \%)$ & 0.34 \\
\hline Days to reversal (range) & $114(84-135)$ & $99(66-163)$ & 0.51 \\
\hline \multicolumn{4}{|l|}{ Operation time, in minutes (range) } \\
\hline resection & $126(113-163)$ & $195(150-255)$ & $<0.0001$ \\
\hline reversal & $177(141-216)$ & $70(60-90)$ & $<0.0001$ \\
\hline $\begin{array}{l}\text { laparoscopic reversal } \\
\text { open reversal }\end{array}$ & $\begin{array}{l}166(137-190) \\
254(175-301)\end{array}$ & & 0.04 \\
\hline LOS resection, days (range) & $17(13-25)$ & $18.5(14-26)$ & 0.38 \\
\hline LOS reversal, days (range) & $10(8-12)$ & $8(6-11)$ & 0.06 \\
\hline
\end{tabular}

Among the patients who underwent PA, $84 \%(n=27)$ suffered from acutely perforated diverticulitis.

\section{Reversal rate}

Reversal could be performed in $75.0 \%$ of patients after HP $(24 / 32)$ compared to in $87.5 \%$ of patients after PA (28/32) ( $p=0.34)$. In strategy A, surgeons (with the patients' consent) refrained from colostomy reversal in seven patients due to severe comorbidity or in one patient for fecal incontinence. Four patients who underwent strategy B did not receive an IC due to transplant organ infection in a kidney transplant patient, severe comorbidity, severe dementia, and a planned neurosurgical intervention. The median time from HP to LHR was 16.3 weeks $(12.0-19.3)$ while the median time from PA to IC was 14.1 weeks $(9.4-23.3)(p=0.51)$.

\section{Morbidity}

The combined major morbidity rate for strategy A was $21.4 \%$ (12 out of 56 procedures) compared to $20 \%$ (12 out of 60 procedures) for strategy $\mathrm{B}(p=1.0)$. The major morbidity rate in strategy A of HP alone was $31.3 \%(n=10)$ as compared to $25.0 \%(n=8)$ after PA with ileostomy in strategy $\mathrm{B}(p=0.78)$. Major morbidity after colostomy reversal were $8 \%(n=2)$ among patients who underwent strategy A, compared to $14.3 \%(n=4)$ among those who underwent strategy B ( $p=$ 0.67) (Fig. 2). Major post-reversal complications in strategy A occurred only in the group of converted patients: one anastomotic leakage and one aspiration pneumonia requiring mechanical ventilation. In strategy $\mathrm{B}$, major post-reversal complications occurred in two patients reoperated for intraabdominal abscess without evidence of anastomotic 
Complications higher than Clavien-Dindo grade IIlb

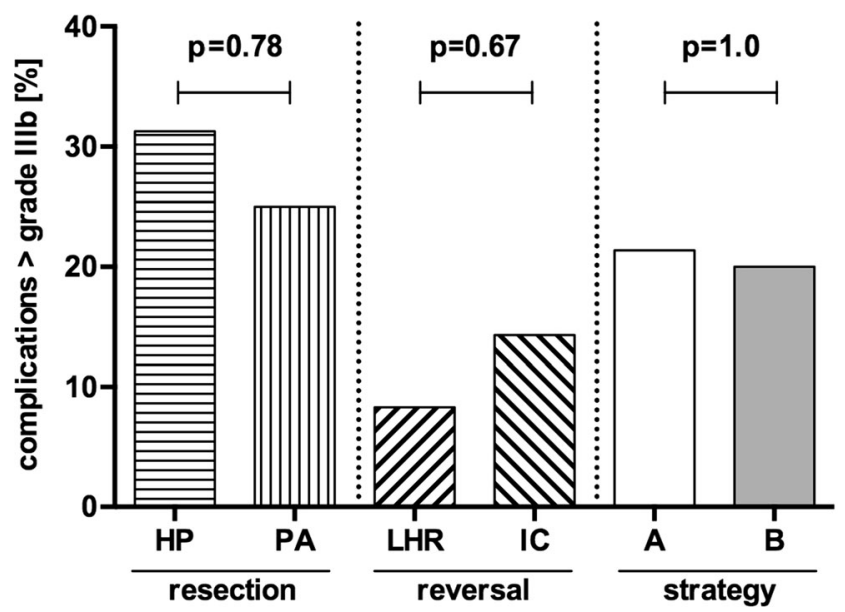

Fig. 2 Major complications (Clavien-Dindo grade IIIb and higher) related to strategy A (HP and LHR), strategy B (PA and IC), and for HP, PA, LHR, and IC. HP Hartmann's procedure, LHR laparoscopic Hartmann's reversal, $P A$ primary anastomosis, $I C$ ileostomy closure

leakage, one patient necessitating wound revision under general anesthesia for wound infection with fascia necrosis. A further patient developed a symptomatic thrombosed aneurysm of the carotid artery and underwent vascular surgical intervention on postoperative day 6 .

The mean CCI, describing the overall morbidity, of strategy A was $16.4 \pm 14.1$, whereas patients receiving strategy B had a CCI of $12.3 \pm 19.1(p=0.08)$. In strategy A, mean CCI for $\mathrm{HP}$ alone was $23.6 \pm 23$ following HP, compared to $16.8 \pm$ 20.7 for PA with loop ileostomy alone in strategy B $(p=0.25)$. The mean CCI associated with LHR was $12.2 \pm 19.9$ compared to $8.8 \pm 20.5$ following IC ( $p=0.33$ ) (Fig. 3).

\section{Comprehensive Complication Index (CCl)}

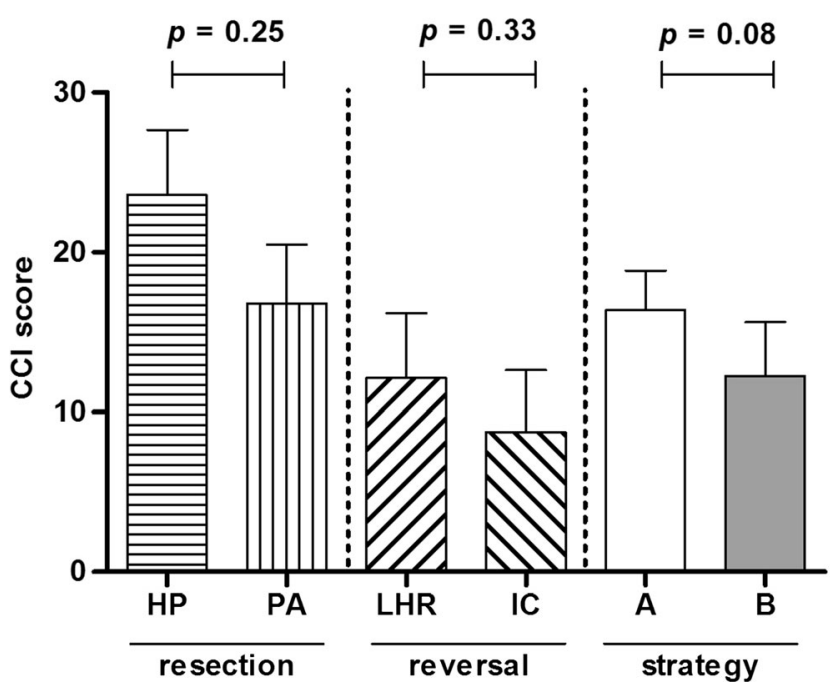

Fig. 3 Comprehensive complication index (CCI) related to strategy A (HP and LHR), strategy B (PA and IC), and for HP, PA, LHR, and IC. HP Hartmann's procedure, LHR laparoscopic Hartmann's reversal, $P A$ primary anastomosis, $I C$ ileostomy closure

\section{Conversion rate}

Although LHR was planned for all HP patients, in 2 patients, it was decided in advance to instead proceed with open reversal for inability to tolerate pneumoperitoneum due to severe cardiopulmonary comorbidity and for large incisional hernia necessitating open repair. Additionally, an attempted LHR in five patients had to be converted to open corresponding to a conversion rate of $22.7 \%$. Reasons for conversion were extensive adhesions $(n=3)$, necessity of mobilizing the right colonic flexure, and short rectal stump. Ultimately, LHR was feasible in 17 out of $24 \mathrm{HP}$ patients (71\%).

\section{Operation time and length of hospital stay}

Table 2 shows the median operation times. Both groups showed similar median lengths of stay for resection. The duration of the second hospital stay for stoma reversal was 10 days (range, $8-12$ days) with strategy A and 8 days (range, $6-11$ days) with strategy $\mathrm{B}(p=0.06)$.

\section{Discussion}

To our knowledge, this is the first case-controlled study to compare the combined morbidity of HP and subsequent LHR (strategy A) to that of sigmoid resection with PA with diverting ileostomy and IC (strategy B) among patients with benign left colonic perforation. We found a high feasibility of LHR and achieved a high stoma closure rate in both groups.

Consistent with retrospective studies [10,11,32], one randomized controlled trial showed a significantly higher reversal rate for PA (90 vs. $57 \%$ ) [16]. However, another randomized controlled trial [17] showed no difference in stoma reversal rates. Our present findings showed a colostomy reversal rate of as high as $75 \%$ despite high comorbidity and ASA scores. We found no disadvantage in terms of the time interval between resection to reversal in strategy A compared to strategy $\mathrm{B}$, which again was in disagreement with current literature [5, 26]. The present high colostomy reversal rate could be explained by the fact that laparoscopic reversal was already planned at time of primary intervention, and patients were followed closely until reversal. Moreover, given the low morbidity and the high surgical feasibility in our experience, the threshold for the indication to LHR was set low. It also has to be taken into account that the favorable results of LHR, which is a technically demanding procedure, were achieved at a certified center of minimally invasive surgery with a high level of laparoscopic expertise and standardization of surgical technique.

This study aimed at comparison of cumulative postoperative major morbidity of the two competing strategies on the basis of an intention-to-treat analysis. Previous studies 
showed lower morbidity associated with IC compared to Hartmann's reversal $[11,16,17,32]$. In this study, we observed a similar major morbidity between the two strategies. Nevertheless, consistent with the literature [11-14, 16, 17], a shorter total operation time, a shorter length of stay after reversal, a trend for lower overall complications, and a trend to higher reversal rates in favor of strategy B were found. PA with ileostomy might still be the better choice when the patient's condition does allow it, and surgical expertise is available. However, it is a reality that experience of the general surgeon on call is limited in a large number of community hospitals thus explaining the sustained frequent use of HP.

\section{Study limitations}

Given the significant variations in the surgical approaches and outcomes following for perforated diverticulitis, the subject of this study is highly relevant. Taken into account the retrospective design and the small sample size, the validity of the conclusions of this study is limited. Due to the limited number of suitable patients, matching had to be restricted to the variables age and comorbidity. However, both groups had similar characteristics as for gender, BMI, and ASA $\geq$ III. Only a larger prospective, preferably randomized controlled trial, could definitively clarify the equality between the two strategies. Yet, both randomized controlled trials [16, 17], which had similar sample sizes as the present study, investigating HP versus PA, had to be terminated prematurely due to poor recruitment. This suggests that an individual rather than a standard strategy is often chosen to deal with this emergency situation among patients with advanced age and multiple comorbidities [3, 33-35]. This conclusion is supported by the fact that in our study, there was a clear trend to a higher ASA score and higher proportion of Hinchey IV in HP. A further limitation of this study is the inhomogeneity of its cohort including not only perforated diverticulitis but also other causes of left-sided colon perforation as anastomotic leakage and ischemia. On the other side, this concerns both comparative groups. The results of the study might be influenced by the fact that HP and PA were performed in different hospitals, and various surgeons were involved. Last, the current study does not elucidate if LHR is superior to open HP reversal, as it does not compare open with laparoscopic HP reversal.

\section{Conclusion}

Since in strategy B, a shorter total operation time, a shorter length of stay after reversal, a trend for lower overall complications, and a trend to higher reversal rates were found, PA with ileostomy might still be the better choice when the patient's condition does allow PA. On the other hand, LHR is feasible in a large proportion of unselected patients with a relatively high reversal rate and moderate morbidity. The present findings suggest that if HP has been performed for colonic perforation, laparoscopic reversal might be considered on the condition of adequate laparoscopic experience. The comparison of the two strategies in this study suggests that by wellperformed LHR, the disadvantages of HP over PA might be at least partially compensated.

\section{Conflicts of interest None}

Authors' contributions DCS, TS, AZ, and AN were responsible for the study conception and design. DCS, TS, PL, and SHL contributed to the acquisition of the data, analysis and interpretation of the data, and drafting of the manuscript. AZ and AN critically revised the manuscript. DCS and TS contributed equally to the study.

\section{References}

1. Regenbogen SE et al (2014) Surgery for diverticulitis in the 21st century: a systematic review. JAMA Surg 149(3):292-303

2. Makela JT, Kiviniemi H, Laitinen S (2005) Prognostic factors of perforated sigmoid diverticulitis in the elderly. Dig Surg 22(1-2): $100-6$

3. Vermeulen J, Lange JF (2010) Treatment of perforated diverticulitis with generalized peritonitis: past, present, and future. World J Surg 34(3):587-93

4. Rafferty J et al (2006) Practice parameters for sigmoid diverticulitis. Dis Colon Rectum 49(7):939-44

5. van de Wall BJ et al (2010) Conventional and laparoscopic reversal of the Hartmann procedure: a review of literature. J Gastrointest Surg 14(4):743-52

6. Maggard MA et al (2004) What proportion of patients with an ostomy (for diverticulitis) get reversed? Am Surg 70(10):928-31

7. Vermeulen $J$ et al (2007) Outcome after emergency surgery for acute perforated diverticulitis in 200 cases. Dig Surg 24(5):361-6

8. Cirocchi R et al (2013) Treatment of Hinchey stage III-IV diverticulitis: a systematic review and meta-analysis. Int J Color Dis 28(4): 447-57

9. Gawlick U, Nirula R (2012) Resection and primary anastomosis with proximal diversion instead of Hartmann's: evolving the management of diverticulitis using NSQIP data. J Trauma Acute Care Surg 72(4):807-14, quiz 1124

10. Zingg U et al (2010) Primary anastomosis vs Hartmann's procedure in patients undergoing emergency left colectomy for perforated diverticulitis. Color Dis 12(1):54-60

11. Breitenstein $\mathrm{S}$ et al (2007) Emergency left colon resection for acute perforation: primary anastomosis or Hartmann's procedure? A casematched control study. World J Surg 31(11):2117-24

12. Constantinides VA et al (2006) Primary resection with anastomosis vs. Hartmann's procedure in nonelective surgery for acute colonic diverticulitis: a systematic review. Dis Colon Rectum 49(7):966-81

13. Abbas S (2007) Resection and primary anastomosis in acute complicated diverticulitis, a systematic review of the literature. Int $\mathrm{J}$ Color Dis 22(4):351-7

14. Salem L, Flum DR (2004) Primary anastomosis or Hartmann's procedure for patients with diverticular peritonitis? A systematic review. Dis Colon Rectum 47(11):1953-64

15. Tabbara $\mathrm{M}$ et al (2010) Missed opportunities for primary repair in complicated acute diverticulitis. Surgery 148(5):919-24 
16. Oberkofler $\mathrm{CE}$ et al (2012) A multicenter randomized clinical trial of primary anastomosis or Hartmann's procedure for perforated left colonic diverticulitis with purulent or fecal peritonitis. Ann Surg 256(5):819-26, discussion 826-7

17. Binda GA et al (2012) Primary anastomosis vs nonrestorative resection for perforated diverticulitis with peritonitis: a prematurely terminated randomized controlled trial. Color Dis 14(11):1403-10

18. Toorenvliet BR et al (2010) Laparoscopic peritoneal lavage for perforated colonic diverticulitis: a systematic review. Color Dis 12(9):862-7

19. Rogers AC et al (2012) Laparoscopic lavage for perforated diverticulitis: a population analysis. Dis Colon Rectum 55(9):932-8

20. Swank HA et al (2013) Early experience with laparoscopic lavage for perforated diverticulitis. Br J Surg 100(5):704-10

21. Netherlands Trial Register. Laparoscopic peritoneal lavage or resection for generalised peritonitis for perforated diverticulitis: a nationwide multicenter randomised trial (The Ladies Trial). 25 April 2014]; Available from: http:/www.trialregister.nl/trialreg/admin/ rctview.asp? $\mathrm{TC}=2037$

22. David GG et al (2009) Use of Hartmann's procedure in England. Color Dis 11(3):308-12

23. Siddiqui MR, Sajid MS, Baig MK (2010) Open vs laparoscopic approach for reversal of Hartmann's procedure: a systematic review. Color Dis 12(8):733-41

24. Zimmermann $\mathrm{M}$ et al (2014) Laparoscopic versus open reversal of a Hartmann procedure: a single-center study. World J Surg

25. Svenningsen PO, Bulut O, Jess P (2010) Laparoscopic reversal of Hartmann's procedure. Dan Med Bull 57(6):A4149

26. Huynh $\mathrm{H}$ et al (2011) Laparoscopic colostomy reversal after a Hartmann procedure: a prospective series, literature review and an argument against laparotomy as the primary approach. Can J Surg 54(2):133-7

27. Arkenbosch J et al (2014) Efficacy of laparoscopic-assisted approach for reversal of Hartmann's procedure: results from the American College of Surgeons National Surgical Quality Improvement Program (ACS-NSQIP) database. Surg Endosc

28. Charlson ME et al (1987) A new method of classifying prognostic comorbidity in longitudinal studies: development and validation. $\mathrm{J}$ Chronic Dis 40(5):373-83

29. Hinchey EJ, Schaal PG, Richards GK (1978) Treatment of perforated diverticular disease of the colon. Adv Surg 12:85-109

30. Dindo D, Demartines N, Clavien PA (2004) Classification of surgical complications: a new proposal with evaluation in a cohort of 6336 patients and results of a survey. Ann Surg 240(2):205-13

31. Slankamenac K et al (2013) The comprehensive complication index: a novel continuous scale to measure surgical morbidity. Ann Surg 258(1):1-7

32. Alizai PH et al (2013) Primary anastomosis with a defunctioning stoma versus Hartmann's procedure for perforated diverticulitis - a comparison of stoma reversal rates. Int J Color Dis 28(12):1681-8

33. Constantinides VA et al (2007) Operative strategies for diverticular peritonitis: a decision analysis between primary resection and anastomosis versus Hartmann's procedures. Ann Surg 245(1):94-103

34. Zorcolo L et al (2003) Toward lowering morbidity, mortality, and stoma formation in emergency colorectal surgery: the role of specialization. Dis Colon Rectum 46(11):1461-7, discussion 1467-8

35. Moore FA et al (2013) Position paper: management of perforated sigmoid diverticulitis. World J Emerg Surg 8(1):55 